selected micronutrient intakes in children across the WHO European Region (WER) using the latest available NDS intakes and WHO Recommended Nutrient Intakes (RNIs) to highlight vulnerable groups and areas of concern.

Methods NDS information was gathered primarily by internet searches and contacting survey authors and nutrition experts. WER countries were grouped into areas - Western, Northern and CEEC. Survey characteristics, reported energy, and nutrient intakes by gender and age group were taken from the latest NDS reports from post-2000 surveys. Population weighted means were calculated and presented by country, European area and WER-wide for both genders and age groups $<10 \mathrm{y}$ and $\geq 10 \mathrm{y}$. WHO RNIs were used to assess nutrient intake adequacy and highlight aspects of concern.

Results Child energy and nutrient intakes were only available from 21 NDS across a third $(n=18)$ of WER countries. Energy and macronutrients, where boys and older children had higher intakes, were more widely reported than micronutrients. Trans fats, omega fats, added sugar and iodine were the least reported nutrients. Countries in all European areas had poor RNI attainment levels; most countries did not meet the carbohydrate, sugar, saturated fat or fibre RNIs in any age group. Older adolescents were more likely to meet RNIs based on absolute levels rather than \%E. Micronutrient attainment was higher than macronutrients, but worst in girls and older children. Iron, vitamin D, folic acid and sodium intakes were of concern, particularly in girls and children aged $\geq 10 \mathrm{y}$. Only six surveys reported intakes by various socio-economic indicators.

Conclusion Only a third, mainly Western, WER countries provided published child nutrient intake data. Gaps in provision mean dietary inadequacies may go unidentified, preventing evidence-based policy formation. WHO RNI attainment was poor, particularly in girls and older children. Inconsistent age groups, dietary methodologies, nutrient composition databases and under-reporting hinder inter-country comparisons. Future efforts should encourage countries to conduct NDS in a standardised format by gender, age and socio-demographic variables. A European-wide policy focus to improve intakes, particularly in girls and children aged $\geq 10 \mathrm{y}$ would be beneficial.

The authors are responsible for the views expressed in this publication and they do not necessarily represent the decisions or stated policy of WHO.

\section{P46 HOW DOES ETHNIC ADJUSTMENT OF CHILDHOOD BMI CHANGE OBESITY PREVALENCE IN INNER LONDON BOROUGHS WITH HIGH ETHNIC DIVERSITY: ANALYSIS USING NATIONAL CHILD MEASUREMENT PROGRAMME DATA FOR 2015-2017} 1J Robson, ${ }^{1} \mathrm{~K}$ Boomla. ${ }^{1}$ Centre for Primary Care and Public Health, Barts and The London School of Medicine and Dentistry, Queen Mary, University London, London, UK; ${ }^{2}$ Public Health Team, London Borough of City and Hackney, London, UK; ${ }^{3}$ Public Health Team, London Borough of Tower Hamlets, London, UK; ${ }^{4}$ Public Health Team, London Borough of Newham, London, UK; ${ }^{5}$ Population Health Research Institute, St George's, University of London, London, UK

10.1136/jech-2018-SSMabstracts. 171

Background for ethnic adjustment of BMI are available but not currently used in the National Child Measurement
Programme (NCMP). We evaluated the effect of ethnic-specific BMI adjustments on the prevalence of obesity and severe obesity, using cut-offs to identify children potentially needing clinical intervention, in three inner London Boroughs with high obesity prevalence and ethnic diversity.

Methods We analysed de-personalised NCMP data for 21126 (10,348 girls) five year-olds $(5,463,9065$ and 6598 respectively in City \& Hackney, Newham and Tower Hamlets) and 19024 (9,361 girls) 11 year-olds (4,865, 8274 and 5,885) for the school years 2015-16 and 2016-17. We estimated, for each borough, the prevalence of obesity (BMI centile $\geq 98$ th) and severe obesity (defined as $\geq 120 \%$ of the 95 th centile) based on unadjusted and ethnic-adjusted BMI using ethnic-specific BMI adjustments for South Asian and Black ethnicity children, developed by Hudda et al. These add $\sim 1.1 \mathrm{~kg} / \mathrm{m}^{2}$ to, and deduct between 0.12 and $5.52 \mathrm{~kg} / \mathrm{m}^{2}$ from, the BMI of South Asian and Black ethnicity children respectively. We report the net change in numbers identified for possible clinical intervention over both school years and for both ages combined.

Results Proportions of five and eleven year-olds of South Asian ethnicity were highest in Tower Hamlets (58.5\%; 67.4\% respectively) and Newham (39.1\%; 30.3\%), and lowest in City \& Hackney $(9.7 \%$; 6.4\%). Equivalent proportions of children of Black ethnicity were highest in City \& Hackney $(29.6 \% ; 22.0 \%)$ and Newham $(16.5 \% ; 13.6 \%)$, and lowest in Tower Hamlets $(8.3 \%$; 9.4\%). Ethnic-specific BMI adjustments increased the respective prevalences of obesity and severe obesity in Tower Hamlets from $6.7 \%$ and $1.7 \%$ to $8.1 \%$ and $2.5 \%$ at age five, and from $11.2 \%$ and $6.9 \%$ to $11.9 \%$ and $8.6 \%$ at age 11 , a net increase of 133 obese and 154 severely obese children. Equivalent prevalences in City \& Hackney fell after ethnic adjustment, from $6.7 \%$ and $1.8 \%$ to $5.2 \%$ and $1.4 \%$ at age five, and from $10.7 \%$ and $8.8 \%$ to $9.4 \%$ and $7.7 \%$ at age 11, a net decrease of 145 obese and 74 severely obese children. Prevalence estimates before and after ethnic adjustment in Newham were broadly similar, resulting in three fewer obese and 28 more severely obese children.

Conclusion Adoption of ethnic-adjusted BMI in ethnically diverse areas alters local estimates of childhood obesity, avoids misclassification of children of South Asian and Black ethnicity, and is essential to support clinical service planning and commissioning. Ethnic-specific BMI adjustments for children of mixed ethnicity are needed.

\section{P47 HIGH PREVALENCE OF OBESITY IN THE GAMBIA: EVIDENCE FROM A NATIONWIDE POPULATION-BASED CROSS SECTIONAL HEALTH EXAMINATION SURVEY}

B Cham*, S Scholes, L Ng Fat, O Badjie, N Groce, JS Mindell. Epidemiology and Public Health, University College London, London, UK

\subsection{6/jech-2018-SSMabstracts. 172}

Background The prevalence of obesity has more than doubled in West Africa over the past 15 years. Obesity is increasing at a faster rate in developing countries compared with developed countries. Possible explanations include epidemiological and nutritional transition, increased consumption of processed foods, and urbanisation. A 1996 study revealed a double burden of over- and under-weight in The Gambia. We examined overweight and obesity prevalence and the associated risk factors in Gambian adults. 